DOI 10.37882/2223-2982.2021.09-2.03

\title{
ГЕЙМИФИКАЦИЯ ОБРАЗОВАТЕЛЬНОГО КОНТЕНТА \\ В УСЛОВИЯХ СМЕШАННОГО ОБУЧЕНИЯ В МЕДИЦИНСКИХ ВУЗАХ
}

\section{GAMIFICATION OF EDUCATIONAL CONTENT UNDER BLENDED LEARNING IN MEDICAL SCHOOLS}

T. Baeva

A. Kuriseva

A. Sitnikova

Summary: The article considers the concept of gamification, its content in the educational settings and the structure of content organization according to its implementation strategy. A review of specific characteristics of gamified content and main principles of its development for the discipline "Foreign language" has been made. The article analyzes LMS Moodle, the most popular learning platform at universities, which maintains generally accepted standards for e-learning. The authors suggest using role-playing game platform Classcraft, that compensates deficiency in gamification of distant learning process, and describe the experimental study based on it. The conclusions demonstrate that gaming activities stimulate visualization of independent learning activities of students, raise their studying motivation due to their involvement to the gaming process and breakdown of psychological barrier that had a positive effect on academic performance of medical students in discipline "Foreign language".

Keywords: e-learning, gamification, LMS Moodle, educational roleplaying game Classcraft.

\author{
Баева Тамара Ахматовна \\ К.n.н., доцент, Северо-Западный государственный \\ медицинский университет \\ им. И.И. Мечникова (Санкт-Петербург), \\ tamarabaeva@mail.ru \\ Курисёва Анна Владимировна \\ ассистент, Северо-Западный государственный \\ медицинский университет \\ им. И.И. Мечникова (Санкт-Петербург) \\ Anna.Kuriseva@szgmu.ru \\ Ситникова Анастасия Андреевна \\ ассистент, Северо-Западный государственный \\ медицинский университет \\ им. И.И. Мечникова (Санкт-Петербург) \\ Anastasiya.Sitnikova@szgmu.ru
}

Аннотация: В статье рассматривается понятие геймификации и ее содержание в условиях системы образования, проанализирована система организации контента по стратегии ее реализации. Сделан обзор специфических характеристик геймифицированного контента и принципов его создания для дисциплины «Иностранный язык». Проводится анализ наиболее распространенной в высшей школе платформы LMS Moodle, которая поддерживает самые популярные стандарты в электронном обучении. Авторы предлагают использовать игровую платформу Classcraft, компенсирующую недостающие средства геймификацирования процесса дистанционного обучения, и описывают экспериментальное исследование на ее базе. По результатам исследования сделан вывод, что игровая деятельность способствует визуализации личной самостоятельной деятельности студентов, их мотивированности к обучению, благодаря вовлеченности в игровой процесс и снятию психологического барьера, что повлияло на улучшение успеваемости студентов медицинского вуза по дисциплине «Иностранный язык».

Ключевые слова: геймификация, смешанное обучение, LMS Moodle, образовательная игровая платформа Classcraft.

месту, темпу усвоения [6,11]. Но, к сожалению, не всегда данная модель компенсирует недостаток очного взаимодействия, как из-за неготовности студентов к самостоятельности и организованности, с одной стороны, так и использования преподавателями таких образовательных технологий, которые могут сделать обучение гарантированно интересным, содержательным и вовлекающим. При этом, одним из инновационных способов стимуляции мотивации к изучению иностранного языка, включающего естественный соревновательный эффект, является геймификация. Целью данного исследования является определение потенциала имеющихся средств дистанционного обучения в медицинском университете с точки зрения геймификации образования и обоснова- 
ние использования ресурсов, возмещающих недостающие элементы.

Рассмотрим геймификацию в рамках «цифровизации», как относительно новый, но уже показавший свою эффективность способ использования игровых технологий и элементов игры в неигровых процессах, в частности, в образовании. Под «цифровизацией» в образовательной среде мы подразумеваем образовательные услуги, которые опосредуются цифровыми технологиями $[7,13]$. Одним из распространенных путей организации материала для эффективного изучения является система компьютерного обучения под названием «Метод четырех дверей», разработанная ученым и консультантом Сивасайламом Тиагараджаном. Он обозначил четыре пути освоения курса, с включением опционального геймификационного контента, соответствующих группам элементов прохождения обучения: библиотека, игровая площадка, кафе, оценочная камера пыток [2]. Обозначаемым эффективным средством создания положительного отклика от реализуемой деятельности, согласно исследования Э. Клопфера, является геймификация, способствующая формированию позитивной реакции на курс и взаимодействию с содержащимся материалом [8,9] путем внедрения игровых элементов в актуальных контекстах для достижения поставленных разработчиками целей [15]. Применительно к образовательной сфере - это подход, предусматривающий применение в учебном процессе принципов компьютерных игр, игровых сценариев и динамик в неигровых ситуациях [1]. Для этой цели внедряется система очков, рейтингов, различные игровые сценарии, создающие у студентов ощущение важности проекта, в котором они принимают участие. Подобный метод подходит при выполнении профильных задач с созданием дополнительного мотива привычной деятельности, по этой причине его использование уместно при преподавании иностранного языка для специальных целей.

Первой «двери» и стратегии овладения курсом присущи изучаемые материалы, второй - интерактивные, игровые упражнения, третьей - социальные игры, подразумевающие наличие соревновательного элемента, четвертой-представляющие серию вопросов, контролирующий уровень понимания. При перерассмотрении «дверей» с точки зрения ведущей направленности В.А. Буров определяет следующие актуальные элементы в геймификации образовательного контента: информирующий, тренировочный, коллаборативно-практический, контролирующий. При этом три из указанных элемента включают элементы игры [4].

Наполнение и формат выделяемых путей обусловлен эффективностью освоения материала, получаемого в различной форме, что продемонстрировано в пирамиде обучения. Наивысшая степень освоения материала приходится на групповое обсуждение, практику конкретной работы, обучение других и использование изученного при решении проблемы. Наименьшую эффективность имеют: лекции и чтение [5]. Это указывает на то, что пассивные формы обучения низкими показателями по запоминанию информации. Именно активные пути освоения нового материала составляют большую часть компьютерного курса, однако пассивный материал присутствует для первичного ознакомления с вопросом и в качестве основы для дальнейшей структуризации и применения.

Актуальные средства организации дистанционного обучения включают возможность создания элементов курса в игровых формах. При обучении студентов медицинского вуза иностранному языку используется одна из наиболее популярных в современных реалиях систем управления учебной деятельности LMS MOODLE модульная объектно-ориентированная динамическая учебная среда). В базовой версии Moodle присутствуют почти все группы элементов информирующий (лекции, глоссарий), тренировочный (задания, тест), контролирующий (тест), коллаборативно-практический (семинар и чат). Исследование потенциала платформы позволило выявить частичное несоответствие требованиям геймификации. При частично неконтролируемом процессе освоения учебного материала, за счет задействования фрагментарно используемых игровых технологий присутствует проблема, заключающаяся в том, что у некоторых студентов отсутствует желание выполнять объем заданий сверх обозначенных как «обязательные». Мы выяснили, что следование актуальному тренду процесса усвоения материала с помощью создания тематической игровой оболочки в цифровой среде сможет стимулировать прохождение всех этапов и, в случае неполучения ожидаемых результатов на конкретном этапе, использовать дополнительные компенсирующие задания элемента.

Принципами создания геймифицированного контента являются:

- наличие компенсаторных действий для достижения цели;

- посильность достигаемых целей;

- последовательное усложнение задач;

- вариативность стратегий;

- визуализация конечной цели и личного прогресса [12].

Из ранее указанного можно отметить, что наличие и вариативность содержательного компонента курса на базовой версии MOODLE обеспечивает основу, однако функционал не снабжает средствами визуализации сопоставления успехов и их поощрения. Предложенный B.А. Стародубцевым вариант коллекционирования монет представляет сложность в калькировании опыта при создании оболочки для дисциплины «Иностранный язык», заключающуюся в невозможности градации успе- 
хов в развиваемых навыках внутри тематического блока, сложности в внедрении рейтинга и вознаграждении победителей [3].

Отличительными характеристиками геймифицированного контента являются: круг пользователей, включающий всех участников образовательного процесса, вознаграждение выполнения заданий, имеющее аккумулирующий эффект, при этом прохождение блоков возможно при достижении установленного рубежа, наличие рейтинговой системы достижений [10].

Задачи, которые должны выполнить обучаемые для перехода на более высокие уровни и получения наград, направлены на овладение навыками и умениями. При этом самостоятельная работа студентов приносит индивидуальные награды, компенсирующие нехватку очков в ранее полученной внутри блока сумме (например, значков) для прохождения барьера или получения лучшего результата. Следует отметить, что коллективная деятельность является ответом на требование общества, по этой причине действия, требующие взаимодействия с другими участниками программы, являются социальным компонентом. Это подчеркивает важность внедрения элемента курса, способствующего взаимной поддержки при освоении материала и совместного пополнения банка знаний, умений и навыков. Выведение социальной основы отдельным блоком предполагает необходимость обеспечения его особым типом наград [16].

Предполагаемые дополнения, а именно многоуровневость системы оценки, включающая степень успешности прохождения блока (уровня), темы, курса, включение тренировочных упражнений в компенсаторный механизм конкретного уровня, выделение отдельного обязательного блока наград социального взаимодействия, визуализация рейтинга группы и индивидуальных успехов, требуют задействования сторонних ресурсов для расширения возможностей программы. Существует довольно широкий и многообразный спектр программных сервисов и платформ, обладающих дидактическим потенциалом для геймификации обучения: Classcraft, MinecraftEdu, LinguaLeo, DuoLingo и др. Среди изученных нами, наиболее подходящей для максимально полного воплощения идеи геймификации мы определили платформу ClassCraft, относящуюся к сфере проектирования обучения и по сути, представляющую собой игровой вариант бально-рейтинговой системы. Classcraft - это разновидность игры живого действия. Она предполагает наличие совместной деятельности и коммуникации, общий предмет и способ деятельности, иерархию внутриигровых мотивов, имитацию реальных процессов [14].

\section{Методы}

Согласно рассмотренным характеристикам и запросам геймификации дисциплины “Иностранный язык” при обучении студентов медицинского вуза инструментов базовой версии MOODLE оказалось недостаточно, по этой причине мы решили прибегнуть к использованию игровой платформы Classcraft для того, чтобы компенсировать ее недостаточность. Мы полагали, что данная платформа могла бы создать дополнительный стимул, влияющий на успеваемость, поведение, мотивированность студентов.

Используя идею Сивасайлама Тиагараджана мы выбрали платформу, отражающую все указанные им компоненты: в начале каждой станции квеста содержатся изучаемые материалы, далее следуют интерактивные, игровые упражнения. Сам формат предполагает наличие визуализированного соревновательного элемента и, безусловно, большое значение имеет контроль усвоения изученного.

При прохождении тем: «Симптомы заболеваний», «Лекарственные препараты», «Альтернативная медицина» использовался ресурс Classcraft. Разработанные нами сценарии и квесты позволили студентам зарабатывать баллы, отвечая на вопросы через призму изучаемой темы. Вовлеченность в соревновательный процесс в команде и индивидуально помогла снизить уровень стресса, сняв психологический барьер.

Наш эксперимент носил краткосрочный характер, количество участников - студентов 1 курса лечебного и медико-профилактического факультетов СЗГМУ им. И.И. Мечникова - составило 100 человек. При проведении практического или семинарского занятия преподавателем выделялись экспериментальная и контрольная группы с приблизительно равным количеством участников. Экспериментальной группе предлагалось самостоятельно выполнить задания с использованием игровой платформы Classcraft, в то время, как контрольная группа имела доступ к тем же заданиям, но вне игровой среды. Установкой к выполнению являлось: «выполнение заданий для отработки материала, полученного на занятии», при этом преподаватель не ориентировал студентов на объем выполненных заданий, т.е. количественная установка отсутствовала. На платформе осуществлялась оценка как командных, так и личных результатов участников эксперимента, а также менялся рейтинг каждого участника в своей команде при реализации проектов и личных выступлений. Система дополнительных поощрений, отличных от уже встроенных в игровую платформу, не разрабатывалась. На платформе использовались только функции базового тарифа.

\section{Результаты}

Для анализа результатов эксперимента использовались следующие критерии: вовлеченность участника в игровой процесс (выполнение заданий с соблюдением критериев и сроков), изменение уровня активности 
участника в ходе эксперимента. Исходя из данных критериев были выделены следующие группы участников:

А. Группы студентов по вовлеченности в игровой процесс:

- Наивысшей вовлеченности (от 90\% выполненных заданий) - $20 \%$

- Высокой вовлеченности (от 75 - 80\%) - 38\%

- Средней вовлеченности (от 60 - 75\%) - 22\%

- Низкой вовлеченности (от 30 - 50\%) - 19\%

- Отсутствующей вовлеченности - 1\% (позиция игнорирования игровых технологий на уроках).

Б. Группы студентов по критерию «изменение уровня активности»:

- уникально-активные - проявившие возросшую активность и заинтересованность в выполнении заданий в рамках самостоятельной деятельности после внедрения игровой технологии;

- регулярно-активные - демонстрирующие активность и заинтересованность в выполнении заданий в рамках самостоятельной деятельности после внедрения игровой технологии аналогичную той, что присутствовала до эксперимента;

- неравномерно-активные - проявившие повышенную активность и заинтересованность в выполнении заданий в рамках самостоятельной деятельности после внедрения игровой технологии на этапе адаптации, пришли к исходному интересу в период индивидуальных заданий и вновь повысили при коллективной деятельности;

- регулярно-пассивные - не проявившие активность, отличающуюся от исходно-пассивной (выполнение немногим более $30 \%$ заданий);

- уникально-пассивные - проявившие позицию отрицания такой формы деятельности (1\% от общего количества участников).

При рассмотрении рейтинга активности (выполнения заданий самостоятельной деятельности студентов на игровой платформе) наивысшую активность проявляют замкнутые, но позитивно настроенные к изучению английского языка студенты (56\% от общего количества участников) - уникально-активные. Их личностно-психологические характеристики при первичном наблюдении: немногословность, отстраненность, отрицание способностей к английскому языку, повышенная эмоциональность при устном общении, также по результатам беседы у них выявлен интерес к компьютерным играм и играм, установленных на мобильных устройствах.

Стабильную активность демонстрировали студенты, входящие в группу регулярно-активных (40\% от общего количества участников). Они продемонстрировали тщательность выполнения всех устных и письменных заданий как при наличии, так и при отсутствии геймификации процесса, однако даже для них такой формат внес изменения в образовательный процесс. Согласно результатам опроса большинству участников (а именно 58\%) стало комфортнее находиться в коллективе и работать в парах и группах, что позволяет сделать предположение о снятии возможных негативных психологических установок и улучшении процесса адаптации студентов среди членов группы.

Переменная активность наблюдалась среди участников, входящих в группу неравномерно-активных студентов (4\% от общего количества). В процессе наблюдения нами было выявлено, что студенты, входящие в данную группу, демонстрируют низкую активность в индивидуальной самостоятельной работе, но при этом высокую активность в групповой работе. Вероятно, это объясняется стремлением студентов сохранить высокий статус в группе, проявлением скрытого лидерства у участников, а также снижением уровня страха совершить ошибку вследствие разделения ответственности за результат среди всех членов группы. Стоит отметить, что в процессе выполнения игрового задания студенты помещаются в воображаемую среду и управляют созданными ими персонажами, что позволяет уменьшить уровень тревожности и сделать образовательный процесс более психологически комфортным.

Представитель группы уникально-пассивных студентов определил свою позицию как борьбу с интернетигровой-зависимостью и нежелание подвергать себя дополнительным искушениям, предпочитая бумажные варианты заданий, которые выполнялись участником выборочно.

В группу высокой вовлеченности были включены уникально-активные (37\%), регулярно-активные (57\%), неравномерно-активные (6\%) студенты.

В группу средней вовлеченности были включены неравномерно-активные (25\%) и регулярно-активные (75\%) студенты.

Группа низкой вовлеченности большей частью представлена регулярно-пассивными студентами, однако в нее также были включены неравномерно-активные студенты. Представляется сложным высчитать точное соотношение обеих категорий из-за особенностей второй из указанных групп, но примерное соотношение составляет 70\% к 30\%, соответственно.

\section{Выводы}

Применение игровых платформ в процессе смешанного обучения сохраняет и развивает вовлеченность студентов в процесс обучения, повышает активность в выполнении практических заданий, мотивирует их к достижению более высоких результатов в обучении и влияет на улучшение успеваемости студентов ме- 
дицинского вуза по дисциплине «Иностранный язык». Такой формат проведения занятий показал, что благодаря возможности снятия психологического барьера при изучении иностранного языка были внесены позитивные изменения в образовательный процесс. Однако, при анализе результатов опроса среди участников эксперимента были выявлены и некоторые недостатки игровой платформы Classcraft: открытие дополнительных страниц при выполнении интерактивных заданий и связанные с этим неудобства, отсутствие возможности просмотра материалов в офлайн режиме в приложении, отсутствие возможности траты накопленных баллов на приобретение дополнительных курсов или расширение игровых опций (например, строительство мира) внутри этой платформы. Исходя из результатов опроса, данная платформа может быть наиболее эффективно использована при самостоятельной деятельности студентов, поскольку игровой формат мотивирует их выполнять задания сверх обязательного уровня для компенсации не- достаточного уровня усвоения материала на очном занятии. Общая удовлетворенность студентов и желание продолжить работу на данной платформе демонстрируют позитивное эмоциональное отношение к подобному формату обучения.

С точки зрения преподавателя достигнут положительный результат, цель эксперимента - визуализация личной самостоятельной деятельности - была реализована успешно. Однако при этом были выявлены профессиональные сложности в проработке балльной системы и индивидуальной оценки участия в коллективной деятельности.

Полученные нами результаты дают возможность предположить, что дальнейшая геймификация процесса обучения иностранному языку в медицинском университете будет способствовать формированию иноязычной профессиональной компетенции.

\section{ЛИТЕРАТУРА}

1. Афонина М.В., Харламова А.С. Контент-анализ понятия «геймификация» // Вестник Алтайского государственного педагогического университета. 2017. - No 3. - C. 46-50.

2. Смирнова Т.Б. Геймификация в системе мотивации и обучения персонала организации. - М.: МГОУ, 2018. - №11-2 (119). - С. $277-281$.

3. Стародубцев, В.А. Ряшенцев И.В. Элементы геймификации в LMS Moodle // Международный научно-исследовательский журнал. - 2017. - № 61(7). C. $98-101$.

4. Буров В.А. Эдьютейнмент и геймификация в обучении иностранному языку: сходства и различия // Вопросы методики преподавания в вузе. - 2020. T. 9. - № 35. - C. 8-17.

5. Cohen L., Manion L., Morrison K. A Guide to Teaching Practice. -London: Routledge. - 2014. - 225 p.

6. Christiansen C., Horn M., Staker H. Is K-12 blended learning disruptive? An introduction to the theory of hybrids. - Clayton Christensen's Institute for disruptive innovation. $-2013 .-48 \mathrm{p}$.

7. Czerniewicz L. MOOC - making and open educational practices. / L. Czerniewicz, A. Deacon, M. Glover, S. Walji. // Journal of Computing in Higher Education. 2017. - №. 29 (1). - 81-97 p.

8. Denny P. The effect of virtual achievements on student engagement. // Proceedings of the SIGCHI conference on human factors in computing systems. - 2013. pp. 763-772.

9. Dicheva D. Gamification in Education: A Systematic Mapping Study. / D. Dicheva, C. Dichev, G. Agre, G. Angelova. // Educational Technology \& Society. - 2015. Vol. 18(3) - pp. 75-88.

10. Kiryakova G., Angelova N., Yordanova L. Gamification in education. // Proceedings of 9th International Balkan Education and Science Conference. - 2014. - pp. 1-5.

11. Ossiannilsson E. Blended learning in continuous vocational training, and continuous professional development: quality concerns. // Technical Innovation in Blended Learning (TIBL) - Strategic Partnerships for vocational education and training project. - 2018. D0I:10.13140/RG.2.2.24573.61927

12. Simoes J., Díaz Redondo R., Vilas A. (2012). A social gamification framework for a K-6 learning platform. // Computers in Human Behavior. - 2013 . - № 29 (2). pp. $345-353$.

13. Swinnerton B. The Unbundled University: Researching emerging models in an unequal landscape. Preliminary findings from fieldwork in South Africa. / B. Swinnerton, M. Ivancheva, T. Coop, C. Perrotta, et al. // Proceedings of the 11th International Conference on Networked Learning. - Zagreb, Croatia. -2018. pp. 218-226.

14. Classcraft. URL: https://www.classcraft.com/ru/ (дата обращения: 12.01.2021).

15. Deterding S., Khaled R., Nacke L., Dixon D. Gamification: Toward a Definition. // CHI 2011 Workshop Gamification: Using Game Design Elements in Non-Game Contexts. - Vancouver. - 2011. URL: http://hci.usask.ca/publications/view.php?id=219 (дата обращения: 20.02.2021).

16. Hsin-Yuan Huang W., Soman D. A Practitioner's Guide To Gamification of Education. - Toronto: Rotman School Of Management - 2013. URL:http://inside.rotman.utoronto.ca/behaviouraleconomicsinaction/files/2013/09/GuideGamificationEducationDec2013.pdf (дата 0бращения: 02.03.2021). 